Martí Peran

$\&$
Diana
Padrón
Alonso

\section{REPENSAR LO PÚBLICO} INTERSECCIONES ENTRE LAS PRÁCTICAS CULTURALES Y EL ESCENARIO COLECTIVO

En las últimas décadas hemos asistido a un creciente desplazamiento de las prácticas artísticas hacía el espacio social con la voluntad de trascender la noción clásica de "arte público" y abrir nuevas zonas de intersección entre las prácticas culturales y el escenario colectivo. Este giro ha provocado una encrucijada fundamental que obliga a las prácticas artísticas con vocación social a pronunciarse respecto a si contribuyen a fortalecer el modelo de cohesión social establecido (un ideal de integración) o, por el contrario, deberían focalizar su interés en la imaginación y ensayo de otros modelos comunales y constituirse así en un ejemplo de posibles prácticas instituyentes. Solo en la resolución de esta disyuntiva, el rol de las propuestas culturales podrá satisfacer sus expectativas más allá de la lógica de la representación y, en su lugar, abrir la posibilidad de transformar o generar nuevos imaginarios para el cuerpo social. Por otra parte, las mutaciones sociales derivadas de los procesos de globalización política, económica y urbana, obligan a las prácticas artísticas a repensar sus horizontes de actuación para abordar desde una perspectiva transdisciplinar 
cuestiones fundamentales como los nuevos formatos de la movilidad social (sujetos, servicios, mercancías, narraciones,...); los nuevos perfiles identitarios (autóctono, residente, turista, extranjero, indocumentado,..); el reconocimiento de los espacios susceptibles de operar como unidad de potencia sociopolítica (barrio, ciudad,...) y los modos de articulación de procesos colaborativos y de cooperación como alternativa al modelo de sujeto autosuficiente promovido por el capital. La complejidad de todas estas cuestiones exige, en primera instancia, una reflexión sobre las metodologías de trabajo capaces de responder a todas estas líneas de actuación.

El volumen 3 de REG/AC configura la aportación del Grupo de Investigación Art Globalization Interculturality (AGI/ART: 2014SGR 1050) a la plataforma de investigación Dispositivos Post. Prácticas artísticas y redes de acción ciudadana en contextos post-crisis - Seminario Expandido promovido por la Fundació Antoni Tàpies y la productora cultural Idensitat, donde investigadores de la Universitat Autónoma de Barcelona, Universitat Politécnica de Catalunya, Universitat Rovira i Virgili, Universitat Ramon Llull, EINA - Centre Universitari de Disseny i Art y Universitat de Barcelona, abordaron diferentes metodologías y modelos de intervención en la esfera pública desde las prácticas artísticas y las ciencias sociales ${ }^{1}$. Algunas de estas contribuciones, como es el caso del texto "El deporte como un artefacto 'hibrido",2, del profesor del Departamento de antropología, filosofía y trabajo social de la Universitat

\footnotetext{
${ }^{1}$ Para obtener más información sobre esta plataforma de investigación, consultar la página web de Idensitat: <http://idensitat.net/es/idbarrio-barcelona/dispositivos-post/912dispositivos-post-seminario-expandido>

${ }^{2}$ Maza Gutiérrez, G. (2015) El deporte como un artefacto "hibrido". Bricolajes para la intervención social, cultural y creativa en el espacio público. Revista de Estudios Globales y Arte Contemporáneo, Vol. 3, pp.85-106
} 
Rovira i Virgili Gaspar Maza Gutiérrez, o el Transition Desing³, que presentan Tania Costa Gómez y Adrià Garcia i Mateu, de EINA - Centre Universitari de Disseny i Art - Universitat Autónoma de Barcelona, han sido recogidas en la Sección Especial de este volumen, junto a la invitación del Forum Permanente del Instituto de Estudos Avançados da Universidade de Sao Paulo y el Grupo de Investigación GIAI/AE de la Universidad Nacional Autónoma de México ${ }^{4}$, los cuales se hallan en procesos de interlocución sobre similares cuestiones.

Del mismo modo, el presente volumen recoge 13 artículos seleccionados a través de convocatoria abierta, correspondientes a investigadores adscritos a centros y universidades internacionales como la Universitat de Barcelona, Universitat Autónoma de Barcelona, Universidad de Málaga, Universidad Miguel Hernández de Elche, Universitat Jaume I Castelló, Universidad de Costa Rica, Universidad Nacional de Córdoba, Universidad Federal de Bahia, Universidad de Los Andes - Venezuela, Universität Giessen, California Lutheran University y University of the Philippines. Esta amplia selección de investigaciones multidisciplinares, se ha llevado a cabo conscientemente para englobar desde diversas perspectivas cuestiones como Esfera Pública / Espacio Público: Metodologías de actuación (Luis Guerra Miranda: "Democracy \& Art, the New World Academy and the Out-ofPlace Public Space of the Inexistent"; Marta Daho: "Fotografías en cuanto espacio público"; Santiago Cao: "La 'Potencia de No' como resistencia al Estado de Bien-estar Común: la Performance como herramienta para propiciar encuentros en los espacios público"; Teo Lagos: “Wake’ de

\footnotetext{
${ }^{3}$ Costa Gómez, T. y Garcia i Mateu, A. (2015) Transition Desing: Investigación y diseño colaborativo para procesos de emancipación ciudadanos. Revista de Estudios Globales y Arte Contemporáneo, Vol. 3, pp. 66-84

${ }^{4}$ Prando, F. (2015) Relato de un viaje a los museos de la ciudad de Río de Janeiro. Revista de Estudios Globales y Arte Contemporáneo, Vol. 3, pp 107-123; y Serrano, L. et al. (2015) arte / contexto / reflexión y acción. Revista de Estudios Globales y Arte Contemporáneo, Vol. 3, pp. 37-65
} 
Maria Thereza Alves: conocimiento y saberes en expansión"), Prácticas Instituyentes (Marcela Knapp: "Interspaces. Cultural journalism and the transformation of the aesthetic world- perception"); Nuevos perfiles identitarios (Alicia Vanessa Nuñez y Rafaela Fiore Urízar: "Punk's Not Dead in East LA: Exploring the East Los Series and It's Use of Locality and Cultural Hybridity for Eastside Catharsis"; Maria Portia Placino: "Contemporary Art Publics: Mapping Philippine Contemporary, Media, Technologies and Publics"; Sergio Villena Fiengo: "Intervenciones intempestivas en Centroamérica. El anti-ceremonial público en la obra de Regina José Galindo"); Nuevos espacios sociopolíticos (Lynda Avendaño Santana: "Toponimias de la relación arte y nuevos medios como arte público en el contexto del Capitalismo Cognitivo"); y Procesos colaborativos y de autogestión (Ilze Gabriela Petroni: "Estrategias de resiliencia: prácticas de gestión autónoma de arte contemporáneo en la Argentina post-crisis de 2001"; Pilar Chauca: "El Flashmob, respuestas artísticas hoy"; Teresa Marín García y Enrique Salom Marco: "Resistencias y apropiaciones. Laboratorios urbanos de experimentación sociocultural en la ciudad de Valencia").

Asimismo, en la sección Miscelánea de la revista, hemos querido recoger aproximaciones recientes a estas cuestiones por parte de investigadores asociados al grupo de investigación Art Globalization Interculturality: Rafael Pinilla se pregunta por una posible culminación de las formas de protesta, y Manuela Frudà incorpora al colectivo ArtDoulas para hablar de su experiencia en torno a las dinámicas del arte público; así como una valoración, a modo de epílogo, de Ramón Parramón, director de la productora cultural Idensitat. 